\title{
Factores de riesgo e índice pronóstico de complicaciones perioperatorias en cirugía bariátrica laparoscópica
}

\author{
Labrada Despaigne A. ${ }^{1}$ \\ 1 Hospital Universitario "General Calixto García”, La Habana, Cuba.
}

Introducción: El paciente obeso que es tratado con cirugía bariátrica laparoscópica es complejo para el anestesiólogo por sus características anatomofuncionales y enfermedades asociadas que presenta. Por lo que es necesario profundizar en el tratamiento anestésico intraoperatorio y posoperatorio inmediato en cuanto a complicaciones perioperatorias e identificación de factores de riesgo, para poder crear escalas de índices pronósticos, con los recursos que se dispone.

Objetivo: Identificar presencia de complicaciones perioperatorias y su relación con factores preoperatorios y conducta anestésica intraoperatoria que puedan constituir indicadores de predicción de complicaciones.

Metodología: Se realizó un estudio analítico, observacional, longitudinal, retrospectivo en 476 pacientes obesos intervenidos quirúrgicamente por gastroplicatura vertical laparoscópica. Para el análisis de los factores pronósticos se utilizó una estrategia univariada y una multivariada. La muestra fue dividida aleatoriamente en dos grupos: grupo de estimación (GE) y grupo de validación (GV). Las variables o potenciales factores de riesgo fueron analizados en cada grupo para demostrar similitud entre los grupos y asegurar que puedan ser comparados. Con el GE se estimaron funciones de regresión logística para los factores que influyen en la aparición de complicaciones. El análisis multivariado se basó en el ajuste de un modelo de regresión logística con todas las variables. La función obtenida permitió determinar la probabilidad de complicarse en función de estas variables y paralelamente estimar la influencia independiente de cada una sobre las complicaciones. Se evaluó la capacidad de predicción del modelo y se comprobó en el grupo de validación.

Resultados: Las complicaciones intraoperatorias y posoperatorias inmediatas fueron pocas. Se lograron identificar 3 factores de riesgo independiente, dados por el estado funcional respiratorio previo, la técnica anestésica utilizada y el tiempo quirúrgico. Se construyó un índice pronóstico de complicaciones que agrupó a los pacientes en riesgo bajo, intermedio y alto de presentar complicaciones.

Conclusiones: La agrupación de factores de riesgo como índice predictor de complicaciones resultó efectiva porque se pudo estimar y validar la capacidad de predicción de complicación. Con la combinación de 11 de las variables explicativas consideradas en la hipótesis como potenciales factores de riesgo para la aparición de complicaciones, se construyó un índice predictivo que permitió clasificar a los pacientes en bajo, mediano y alto riesgo. El índice construido fue validado en una muestra diferente a la estimada, con un buen poder de discriminación.

https://doi.org/10.25237/congresoclasa2019.12 